\title{
Colorectal emergency surgery in a COVID-19 referral hospital during the phase 2 in Italy
}

\author{
Giorgio Lisi ${ }^{1}$ (D) M. Campanelli ${ }^{2} \cdot$ M. Villa ${ }^{3} \cdot$ S. Grande ${ }^{3} \cdot$ M. Grande ${ }^{3}$
}

Accepted: 25 June 2020 / Published online: 1 July 2020

(C) Springer-Verlag GmbH Germany, part of Springer Nature 2020

Dear Editor:

The outbreak of SARS-CoV-2 infection, first reported in China in December 2019, has become a public health emergency of international concern that also affects patients with colorectal disease [1]. Recently, the University Hospital of Rome Tor Vergata was named "Covid Hospital-4," the fourth referral center in Rome for COVID-19-positive patients. As a result, all surgical services across the department, with the exception of the endoscopic session for oncological and urgent cases, were locked with the result that the vast majority of surgeons operated only in urgent cases (73.9\%) [2], but the number of interventions significantly dropped. In the past 2 weeks, the Italian government has declared "phase 2" of the lockdown reducing restrictions and access to hospitals as well as outpatient. Since then, up to $40 \%$ of non-traumatic abdominal emergencies have had unusual delayed treatment [2]. We share some considerations about our first 2 weeks of colorectal emergency surgery during phase 2 , with particular attention to the increase number of complex colorectal emergency cases. We reported 11 difficult cases of emergency colorectal surgery in contrast to the same period before and during the lockdown: 4 cases of Hinchey III and IV diverticulitis, 3 locally advanced colorectal cancers with intestinal obstruction, and four obstructive right colon cancer. According to the recent survey by Patriti et al. sent to 150 Italian heads of General Surgery [2], about $40 \%$ of surgeons reported an unusual delay in the presentation of non-traumatic

Giorgio Lisi

giolimas06@yahoo.it

1 University Hospital of Tor Vergata, viale Oxford 81, 00133 Rome, Italy

2 Mininvasive Surgery Unit, University Hospital of Tor Vergata, viale Oxford 81, 00133 Rome, Italy

3 Emergency Surgery Unit, University Hospital of Tor Vergata, viale Oxford 81, 00133 Rome, Italy abdominal emergencies. In all cases, the fever had been present for several days before hospitalization. The delay was partially related to patient choice, preferring to stay at home until symptoms worsened, and in part due to the waiting list for the COVID-19 emergency room test, almost half of the centers reported emergency associated with a more serious presentation due to a diagnostic delay. In our country, many patients with fever have asked the authorities not to go to the hospital if they do not have breathing difficulties. In these patients, fever may not be caused by COVID-19-related pneumonia but by an abdominal infection. There are also numerous patients whose diagnostic delay is related to the time spent in the emergency room, both because of the scarcity of hospital beds available and because of the diagnostic work for COVID-19. Our preliminary experience recognizes some of the main reasons for delayed diagnosis during the COVID-19 pandemic. The first is the concern of parents; families were extremely concerned about the possibility of contracting COVID-19 in public places such as outpatient or ER. Caregivers were reluctant to contact their primary care physician, and even after a specific referral to the emergency room, they chose to refrain from visiting medical facilities. The second reason is the inadequate clinical evaluation and the consultation settings; in the COVID-19 era, many of the medical activities were conducted through a telemedicine platform that allows patients to be assessed by limiting exposure [3]. However, one of these disadvantages is the inability to conduct a complete physical examination which can lead to an incorrect diagnosis. We are convinced that this could be the beginning of a worse scenario; in a recent snapshot on behalf of the COVID-SURGE-ITA group [4] conducted on 54 surgical units in 36 Italian hospitals, the survey showed how surgical oncology has suffered a significant reduction in activity resulting in a double waiting list, and the hub-and-spoke cancer program did not work adequately, so we could probably see a dramatic increase in primary symptoms related to cancer, and more patients may require an emergency approach with a significant worse prognostic impact in the future. 
Whatever the reason why these patients or those of others delay the search for medical and surgical care during this pandemic, the concern remains that this delay will lead to an increase in morbidity and mortality in the coming weeks and months. Perhaps in the future, we will see the real consequences on screening programs, and the effect of the COVID-19 pandemic on our population's non-COVIDrelated health could be even more devastating than COVID19 itself.

Authors' contributions GL, MC, MV, SG, and MG contributed equally to this work: conception, design, and drafting the work, and final approval of the version to be published.

\section{Compliance with ethical standards}

Conflict of interest The authors declare that they have no conflicts of interest.

\section{References}

1. Wu Z, McGoogan JM (2020) Characteristics of and important lessons from the coronavirus disease 2019 (COVID-19) outbreak in China: summary of a report of 72314 cases from the Chinese center for disease control and prevention. JAMA. https://doi.org/10.1001/ jama.2020.2648

2. Patriti A, Baiocchi GL, Catena F, Marini P, Catarci M, FACS on behalf of the Associazione Chirurghi Ospedalieri Italiani (ACOI) (2020) Emergency general surgery in Italy during the COVID-19 outbreak: first survey from the real life. World J Emerg Surg 15:36

3. Keesara S, Jonas A, Schulman K (2020) Covid-19 and health care's digital revolution. N Engl J Med 382(23):e82

4. Torzilli G, Vigano L, Galvanin J et al (2020) A snapshot of elective oncological surgery in Italy during COVID-19 emergency: pearls, pitfalls, and perspectives. Ann Surg. https://doi.org/10.1097/SLA. 0000000000004081

Publisher's note Springer Nature remains neutral with regard to jurisdictional claims in published maps and institutional affiliations. 